\title{
Parametric Landau damping of space charge modes
}

\author{
Alexandru Macridin, Alexey Burov, Eric Stern, James Amundson, and Panagiotis Spentzouris \\ Fermilab, P.O. Box 500, Batavia, Illinois 60510, USA
}

(Received 23 September 2016; revised manuscript received 2 May 2017; published 29 January 2018)

\begin{abstract}
Landau damping is the mechanism of plasma and beam stabilization; it arises through energy transfer from collective modes to the incoherent motion of resonant particles. Normally this resonance requires the resonant particle's frequency to match the collective mode frequency. We have identified an important new damping mechanism, parametric Landau damping, which is driven by the modulation of the mode-particle interaction. This reveals new possibilities for stability control through manipulation of both particle and mode-particle coupling spectra. We demonstrate the existence of parametric Landau damping in a simulation of transverse coherent modes of bunched accelerator beams with space charge.
\end{abstract}

DOI: 10.1103/PhysRevAccelBeams.21.011004

\section{INTRODUCTION}

Landau damping (LD) [1] gives rise to the stabilization of collective modes in plasma and accelerator beams. The damping is caused by the energy transfer from the collective mode to the particles in resonance with the mode. The damping rate is, therefore, determined by the number of the particles capable of resonating with the mode. Conventionally, Landau damping requires the coherent resonance frequency to lie within the incoherent spectrum, i.e., to be located within the continuous frequency spectrum of the individual particles. Manipulation of the incoherent spectrum is often proposed as a mean to enhance stability; for example, the increase of the betatron tune spread in accelerators by using nonlinear magnets [2]. In this paper we discuss a new mechanism for Landau damping which occurs when the mode-particle coupling has an extended frequency spectrum. This mechanism reveals new possibilities for stability enhancement involving both particles' and the modeparticle coupling's spectra.

The novel Landau damping mechanism, which we call parametric Landau damping, is revealed by the numerical investigation of the transverse space charge modes in accelerator bunched beams at the coupling resonance, i.e., when the horizontal and the vertical tunes are close. In contrast with the usual Landau damping mechanism, the frequency of the LD-responsible particles, i.e., the particles which absorb the mode energy, have a wide spread and do not match the coherent frequency. This happens due to the

Published by the American Physical Society under the terms of the Creative Commons Attribution 4.0 International license. Further distribution of this work must maintain attribution to the author(s) and the published article's title, journal citation, and DOI. modulation of the mode-particle coupling by particle dependent oscillations with a wide frequency spread.

The transverse space charge modes in bunched beams, away from the coupling resonance were found analytically in Refs. [3-5]. Their intrinsic Landau damping in the strong space charge regime was suggested in Ref. [4]. Predicted damping rates were confirmed by numerical simulations [6-8].

The effect of linear coupling resonance on Landau damping was addressed by Metral in [9]. He showed that in the proximity of linear coupling resonance, the incoherent frequency spectrum from both transverse planes contributes to Landau damping. The collective mode transfers energy to the incoherent motions in both planes. Metral's mechanism is a shared damping between the transverse planes; a damping increase of the modes present in one plane implies a compensating decrease in the damping of the modes present in the other plane. In contrast, our mechanism is due to an oscillating mode-particle coupling implying additional conditions for the resonant energy transfer. Since our mechanism does not involve sharing, it is possible to enhance Landau damping for modes present in both planes. In our case the coupling between the transverse planes is produced by the space charge force, thus no linear terms are present. The main resonance is the fourth-order Montague resonance [10]. In our simulations the frequency of many LD-responsible particles does not match the coherent frequency. The enhancement of the damping rate cannot be explained by Metral's coupling resonance mechanism which requires the presence of resonant particles around the coherent frequency in the plane perpendicular to the mode.

A common feature of the particles trapped in the vicinity of resonance fixed points is the oscillation of their amplitudes. In the coupling resonance case the trapped particles are characterized by an oscillatory energy exchange between the transverse planes. Their transverse amplitudes 
are oscillating with typical trapping frequencies. Since the mode-particle coupling is dependent on the particles' amplitudes, it is modulated by the trapping frequency. The resonance condition for Landau damping requires the particle frequency to equal the mode frequency shifted by the trapping frequency. Because the trapping frequencies are particle dependent, the frequencies of the LD-responsible particles may span a range equal to the one of the modeparticle coupling frequency spectrum.

We employ the Synergia accelerator modeling package $[11,12]$ to simulate the propagation of a single Gaussian beam through a linear lattice. The modes are extracted from the transverse displacement density using the dynamic mode decomposition (DMD) technique [13-16]. DMD is a data driven algorithm used for modal analysis in both linear and nonlinear systems.

We compare the properties of the first space charge mode away and in the vicinity of the coupling resonance. We find that the Landau damping is larger in the latter case, while the frequency and the mode shape are nearly the same. By investigating the properties of the particles exchanging energy with the mode, we conclude that the off-resonance case well fits the conventional Landau damping scenario characterized by LD-responsible particles with an incoherent frequency spectrum at the coherent frequency. Around coupling resonance the damping enhancement is caused by the parametric Landau damping mechanism, a consequence of the modulated coupling between the mode and the trapped particles. The existence of the parametric Landau damping mechanism for the first space charge mode at coupling resonance is proven solely by numerical simulations of a bunch propagating through a lattice; no analytical model is assumed.

The paper is organized as follows. In Sec. II the mechanism of parametric Landau damping is formally introduced. In Sec. III the Landau damping mechanism of transverse space charge modes at coupling resonance is discussed. The details of the simulations are described in Sec. IV. The results of the simulations are presented in Sec. V, followed by discussions in Sec. VI. Summary and conclusions are given in Sec. VII. In Appendix A the calculation of tune shift and mode-particle coupling is discussed.

\section{PARAMETRIC LANDAU DAMPING}

The Landau damping mechanism results from the interaction of the collective mode with the individual particles. Using the simple harmonic oscillation approximation [17], the equation of motion for the particle $i$ interacting with the mode $\bar{x}$ can be written as

$$
\ddot{x}_{i}+\omega_{i}^{2} x_{i}=-K_{i} \bar{x}(t),
$$

where $x_{i}$ represents the particle displacement, $\omega_{i}$ the particle frequency, $K_{i}$ the mode-particle coupling and $\bar{x}(t)$ the collective mode.
In systems with conventional Landau damping, $K$ is either time independent or its oscillation frequency is particle independent. The resonance condition is $\omega_{i}=\omega_{c}$, where $\omega_{c}$ is the $\bar{x}(t)$ frequency, i.e., $\bar{x}(t) \propto \exp \left(-i \omega_{c} t\right)$. The damping rate is proportional to the spectral density at the resonant frequency,

$$
\lambda \propto \rho\left(\omega_{c}\right)=\sum_{i} \delta\left(\omega_{i}-\omega_{c}\right)
$$

Nevertheless, as in the case addressed in this paper, it may happen that the mode-particle coupling is characterized by a frequency spectrum, i.e., $K_{i}(t) \propto \exp \left(-i \mu_{i} t\right)$ and $\mu_{i}$ is particle dependent. The resonance condition in this case is $\omega_{i}=\omega_{c} \pm \mu_{i}$. The damping rate is proportional to the number of particles which fulfill the resonance condition,

$$
\lambda \propto h\left(\omega_{c}\right)=\sum_{i} \delta\left(\omega_{c}-\omega_{i} \pm \mu_{i}\right) .
$$

In this case the damping is determined by the interplay of both particles and mode-particle coupling spectra. We call this mechanism parametric Landau damping.

\section{LANDAU DAMPING OF TRANSVERSE SPACE CHARGE MODES}

For transverse space charge modes the equation of motion for the particle $i$ can be written as (see Appendix A, Eq. (A16), [3-5,18])

$$
\begin{aligned}
& \ddot{x}_{i}+\omega_{0}^{2}\left[Q_{0 x}-\delta Q_{x}\left(z_{i}, J_{x i}, J_{y i}\right)\right]^{2} x_{i} \\
& =-K\left(z_{i}, J_{x i}, J_{y i}\right) \bar{x}\left(t, z_{i}\right),
\end{aligned}
$$

where $\omega_{0}$ is the angular revolution frequency and $Q_{0 x}$ is the bare betatron tune. The tune shift $\delta Q_{x}\left(z_{i}, J_{x i}, J_{y i}\right) \ll Q_{0 x}$ and the mode-particle coupling $K\left(z_{i}, J_{x i}, J_{y i}\right)$ are proportional to the longitudinal density and are dependent on the particle transverse actions, $J_{x i}$ and $J_{y i}$, defined as

$$
\begin{aligned}
& J_{x i}=\frac{x_{i}^{2}+\left(\alpha_{x} x_{i}+\beta_{x} x_{i}^{\prime}\right)^{2}}{2 \beta_{x}}, \\
& J_{y i}=\frac{y_{i}^{2}+\left(\alpha_{y} y_{i}+\beta_{y} y_{i}^{\prime}\right)^{2}}{2 \beta_{y}},
\end{aligned}
$$

where $\alpha_{x}, \beta_{x}, \alpha_{y}$ and $\beta_{y}$ are the lattice Twiss parameters.

The coherent driving term $\bar{x}\left(t, z_{i}\right)$ in Eq. (4) is the average displacement at the location where the particle is currently located and can be written as $\bar{x}\left(t, z_{i}\right)=$ $e^{-i \omega_{0} \nu t} \bar{x}\left[z_{i}(t)\right]$, where

$$
\bar{x}(z)=\frac{\int d x d x^{\prime} d y d y^{\prime} d u x \rho\left(x, x^{\prime}, y, y^{\prime}, z, u\right)}{\rho(z)},
$$


and $\nu$ being the mode tune [4]. Here $z$ is the longitudinal position relative to the reference particle, $u=\frac{\delta p}{p}$ is the relative momentum spread, $\rho\left(x, x^{\prime}, y, y^{\prime}, z, u\right)$ is the density in $6 D$ phase space and $\rho(z)=\int d x d x^{\prime} d y d y^{\prime} d u \rho\left(x, x^{\prime}, y, y^{\prime}\right.$, $z, u)$ is the longitudinal density. Unlike the situation described by Eq. (1), in Eq. (4) the uncoupled particle motion, the mode particle coupling and $\bar{x}$ are not characterized by a single frequency.

In order to address the Landau damping mechanism in the following part of this section we will investigate the conditions for resonant coupling between the particles and the collective mode. The explicit dependence of the tune shift $\delta Q_{x}$ and the mode-particle coupling $K$ on the particle's action coordinates is not important for this analysis.

In the off-resonance case, to a good approximation the particle transverse actions $J_{x i}$ and $J_{y i}$ are constants of motion and the time dependence of the tune shift $\delta Q_{x}\left(z_{i}, J_{x i}, J_{y i}\right)$ is a consequence of the synchrotron motion given by $z_{i}(t)=a_{i} \cos \left(\omega_{0} Q_{s} t+\varphi_{i}\right)$. Here $a_{i}$ is the particle's longitudinal amplitude, $Q_{s}$ is the synchrotron tune and $\varphi_{i}$ is the particle's phase at $t=0$. The tune shift $\delta Q_{x}\left(z_{i}, J_{x i}, J_{y i}\right)$ for a Gaussian beam with the longitudinal density $\rho(z)=\exp \left(-z^{2} / 2 \sigma_{z}\right)$ can be expanded as

$$
\begin{aligned}
& \delta Q_{x}\left[z_{i}(t), J_{x i}, J_{y i}\right] \\
& =\delta Q_{x}\left[0, J_{x i}, J_{y i}\right] \exp \left(\frac{-z_{i}^{2}(t)}{2 \sigma_{z}^{2}}\right) \\
& =\delta Q_{x}\left[0, J_{x i}, J_{y i}\right] \exp \left(\frac{-\left[a_{i} \cos \left(\omega_{0} Q_{s} t+\varphi_{i}\right)\right]^{2}}{2 \sigma_{z}^{2}}\right) \\
& =C_{0 i}+\sum_{m=1}^{\infty} 2 C_{m i} \cos \left[2 m\left(\omega_{0} Q_{s} t+\varphi_{i}\right)\right],
\end{aligned}
$$

where

$$
C_{m i}=\delta Q_{x}\left[0, J_{x i}, J_{y i}\right](-1)^{m} \exp \left(-\frac{a_{i}^{2}}{4 \sigma_{z}^{2}}\right) I_{m}\left(\frac{a_{i}^{2}}{4 \sigma_{z}^{2}}\right),
$$

and $I_{m}$ are the modified Bessel functions of the first kind. The tune shift contains a constant term which depends on the particle's longitudinal amplitude $a_{i}$ and higher harmonics of $2 Q_{s}$ terms. Since the mode-particle coupling $K\left(z_{i}, J_{x i}, J_{y i}\right)$ is also proportional to the charge density $\rho(z)$, an analogous expansion is valid for the mode-particle coupling term

$K\left[z_{i}(t), J_{x i}, J_{y i}\right]=K_{0 i}+\sum_{m=1}^{\infty} 2 K_{m i} \cos \left[2 m\left(\omega_{0} Q_{s} t+\varphi_{i}\right)\right]$.

The mode $\bar{x}\left(t, z_{i}\right)$ frequency as seen by the particle $i$, is also influenced by the synchrotron motion. For that reason, the main tune of the first space charge mode is $\nu-Q_{s}$ and not $\nu$. This can be understood by considering the approximation $\bar{x}[z] \approx \sin \left[\pi z / 4 \sigma_{z}\right][3,6]$ for the first space charge mode. The particle $i$ sees the mode as

$$
\begin{aligned}
\bar{x}\left[t, z_{i}(t)\right] \approx & e^{-i \omega_{0} \nu t} \sin \left[\frac{\pi a_{i} \cos \left(\omega_{0} Q_{s} t+\varphi_{i}\right)}{4 \sigma_{z}}\right] \\
= & e^{-i \omega_{0} \nu t}\left[J_{1}\left(\frac{\pi a_{i}}{4 \sigma_{z}}\right) \cos \left(\omega_{0} Q_{s} t+\varphi_{i}\right)\right. \\
& + \text { higher harmonics }] .
\end{aligned}
$$

Here $J_{1}$ is the Bessel function of the first kind and higher harmonics represent $(2 n+1) Q_{s}$ higher harmonic terms proportional to higher order Bessel functions. They are smaller in magnitude compared to the $J_{1}$ term.

From Eqs. (4), (7), (9), and (10) one can conclude that, in the off-resonance case, the main energy resonant exchange specific to the Landau damping mechanism between the first space charge mode and the particles takes place at the tune $\nu-Q_{s}$. Resonant exchanges at tunes distanced from $\nu-Q_{s}$ by harmonics of $2 Q_{s}$ are also present, but we find that they play only a minor role in the Landau damping mechanism, as discussed in Sec. V.

In the off-resonance case the Landau damping is conventional, since the oscillation frequencies of the mode-particle coupling are particle independent $[K$ contains a constant term and harmonics of $2 Q_{s}$, see Eq. (9)]. The situation is different at the coupling resonance. Unlike the offresonance case, in the proximity of the coupling resonance $J_{x i}$ and $J_{y i}$ are not constants of motion. In fact the sum $J_{s i}=J_{x i}+J_{y i}$ is a constant of motion, while the difference $J_{d i}=J_{x i}-J_{y i}$ oscillates around the stable point with the trapping frequency $\omega_{t i}=\omega_{0} Q_{t i}$ [see Eq. (A42)]. The essential feature for the parametric Landau damping mechanism is that the particle dependent $Q_{t i}$ modulates the mode-particle coupling. This becomes evident when the mode-particle coupling is written as [see Eq. (A54)]

$$
K\left(z_{i}, J_{x i}, J_{y i}\right)=A\left(z_{i}, J_{s i}\right)+B\left(z_{i}, J_{s i}, J_{d i}\right) J_{d i} .
$$

The terms $A\left(z_{i}, J_{s i}\right)$ and $B\left(z_{i}, J_{s i}, J_{d i}\right)$ as well as the tune shift $\delta Q_{x}\left(z_{i}, J_{s i}, J_{d i}\right)$ depend on the beam shape and can be approximated analytically for certain cases. We discuss the calculation of the tune shift and the mode-particle coupling in Appendix A. In the Appendix A 2, to give an example, we calculate the particles' dynamics in the proximity of the coupling resonance stable point for a 2 dimensional (2D) Hamiltonian with a fourth order, rotationally symmetric transverse coupling term. Equation (A54) gives the modeparticle coupling in our example. A more general Hamiltonian describing the dynamics at coupling resonance, but without the presence of collective modes, was considered by Montague [10]. 
The equation of motion in the proximity of the coupling resonance is

$$
\begin{aligned}
& \ddot{x}_{i}+\omega_{0}^{2} Q_{x}^{2}\left(z_{i}, J_{s i}, J_{d i}\right) x_{i} \\
& =-A\left(z_{i}, J_{s i}\right) \bar{x}\left(t, z_{i}\right)-B\left(z_{i}, J_{s i}, J_{d i}\right) J_{d i} \bar{x}\left(t, z_{i}\right),
\end{aligned}
$$

where $Q_{x}\left(z_{i}, J_{s i}, J_{d i}\right)=Q_{0 x}-\delta Q_{x}\left(z_{i}, J_{s i}, J_{d i}\right)$. Since $J_{s i}$ is a constant of motion, the coupling term $A \bar{x}$ is conventional. The term $B J_{d} \bar{x}$ yields parametric Landau damping because it is modulated by oscillations with $\omega_{0} Q_{t i}$ frequency. While in the former case the resonance condition is $\bar{Q}_{x i}=\nu-Q_{s}$, the $B J_{d} \bar{x}$ term implies mode-resonant particles when $\bar{Q}_{x i}=\nu-Q_{s}-Q_{t i}$. Here $\bar{Q}_{x i}$ is the particle $i$ main tune defined as the tune of the largest peak in the Fourier spectrum of $x_{i}(t)$. In principle the dependence of $B\left(z, J_{s}, J_{d}\right)$ on $J_{d}$ yields resonant energy exchanges at frequencies spaced by harmonics of $Q_{t}$ from the main parametric resonant condition, i.e., $\bar{Q}_{x i}=\nu-Q_{s}-Q_{t i}+n Q_{t i}$, but in our simulations we find these processes not to be significant.

The oscillations of $J_{d}$ contribute not only to the parametric Landau damping but to the conventional one as well. The dependence of $Q_{x}\left(z_{i}, J_{s i}, J_{d i}\right)$ in Eq. (12) on $J_{d i}$ yields satellite features spaced by harmonics of $Q_{t i}$ in the incoherent spectrum. These satellites are resonant via $A \bar{x}$ coupling when their tune is equal to $\nu-Q_{s}$.

\section{SIMULATIONS}

The simulations are done by employing SYNERGIA [11], a particle tracking code for beam dynamics in accelerators. The space charge effects are implemented in SYNERGIA using the second order split-operator method [19]. At every step, the electric field is calculated by solving the 3D Poisson equation with open boundary conditions numerically as described in [20].

The bunch is initially excited in the horizontal plane with the first space charge harmonic function. Space charge harmonics are the space charge modes of Gaussian beams in the strong space charge limit and were calculated analytically in Refs. [4,5]. The excitation amplitude is small enough to ensure linear damping regime and not to affect the particles' tune spectrum. The transverse displacement density,

$$
X(z, u, s)=\frac{\int d x d x^{\prime} d y d y^{\prime} x \rho\left(x, x^{\prime}, y, y^{\prime}, z, u, s\right)}{\rho(z, u, s)},
$$

is calculated at every turn. Here $s$ is the distance along the reference trajectory, and

$$
\rho(z, u, s)=\int d x d x^{\prime} d y d y^{\prime} \rho\left(x, x^{\prime}, y, y^{\prime}, z, u, s\right)
$$

is the density in the longitudinal phase space. The modes' shape, tune, and damping are extracted from $X(z, u, s)$ using the DMD technique. DMD [13-16] has been used for mode analysis in many fields such as fluid mechanics [21], neuroscience [22], and video streaming and pattern recognition [23]. An important advantage of this method is the direct calculation of mode dynamics, including shape, frequency, and growth/damping rates. Application of SYNERGIA and DMD to beam dynamics is described in detail in [8].

A lattice with the length $200 \mathrm{~m}$ made by 10 identical OFORODO (drift—focusing quad-drift—rf cavitydrift—defocusing quad-drift) cells is chosen. The difference between the actual phase advance and the smooth approximation phase advance nowhere exceeds $2 \%$ of the phase advance per cell. For the off-resonance case we take the bare betatron tune difference $Q_{0 x}-Q_{0 y}>\delta Q_{s c \max }$ while at the coupling resonance $Q_{0 x}=Q_{0 y} . \delta Q_{s c \max }$ is the space charge tune shift at the center of the bunch. The tunes in our simulations have values in the range typical for real circular accelerators, with $Q_{s} \ll Q_{0 x}, Q_{0 y}$. The majority of the simulations are done with $Q_{0 x}=2.322$ and $Q_{s}=0.01$. We checked the robustness of our results for other values of the tunes, such as $Q_{0 x}=2.443$ and $Q_{s}=0.005$. A proton beam with the energy corresponding to the relativistic factor $\gamma=1.6$ is chosen. The chromaticity is zero. $10^{8}$ macroparticles per bunch are used for the simulations. The beam distribution is longitudinally and transversely Gaussian with equal vertical and horizontal emittances, $\epsilon_{x}^{\mathrm{rms}}=\epsilon_{y}^{\mathrm{rms}}=1 \mathrm{~mm} * \mathrm{mrad}$. The space charge parameter is defined as $q=\frac{\delta Q_{s c \max }}{Q_{s}}$.

\section{RESULTS}

While the formalism described in Secs. II and III and Appendix A is useful for understanding the damping mechanism and interpreting the simulations, the results presented in this section are based only on the tracking simulations of a Gaussian beam through an OFORODO lattice, as described in Sec. IV.

The properties of the first space charge mode offresonance and at coupling resonance are compared in Fig. 1. For intermediate and large space charge, $q \gtrsim 4$, the damping at coupling resonance is larger, as shown in Fig. 1 (a). In the strong space charge regime, $10 \lesssim q \lesssim 20$, the damping at coupling resonance is larger by approximately a factor of 2 . The relative enhancement increases slowly with $q$. However the precision of the simulation at large $q$ is limited by the small value of the Landau damping, which becomes of the order of the error bars. The mode tune measured relative to the bare betatron tune, $\nu-Q_{0 x}$, is nearly the same for both cases, see Fig. 1(b). The difference between the mode spatial shape in the two cases is also insignificant, as illustrated in Fig. 1(c) where the spatial overlap of the mode, $X_{1}(z, u)$, with the first space charge harmonic $Y_{1}(z)\left(Y_{1}(z)\right.$ is calculated in [4]), 


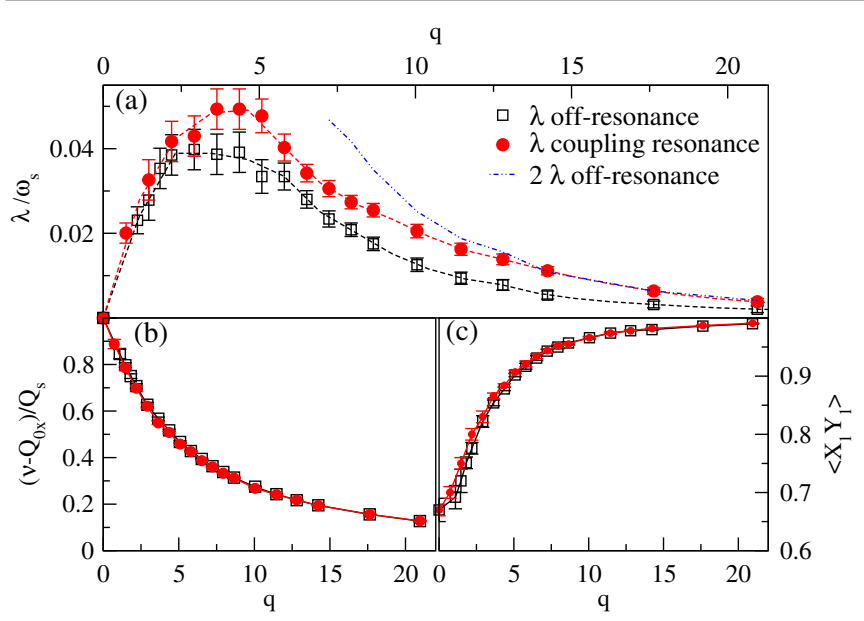

FIG. 1. Comparison between the off-resonance and the coupling resonance cases for the first space charge mode: (a) Landau damping rate $\frac{\lambda}{\omega_{s}}$ versus space charge parameter $q$, where $\omega_{s}=$ $\omega_{0} Q_{s}$ is the synchrotron frequency. For intermediate and strong space charge the damping is larger at the coupling resonance. (b) The mode tune $\nu$ at off-resonance and at coupling resonance are nearly the same. (c) The spatial overlap, Eq. (15), of the DMD extracted mode $X_{1}(z, u)$ with the space charge harmonic function $Y_{1}(z)$. The off-resonance and the coupling resonance mode shapes are nearly the same.

$$
\left\langle X_{1} Y_{1}\right\rangle=\int X_{1}(z, u) Y_{1}(z) \rho(z, u) d z d u
$$

is plotted.

The off-resonance Landau damping mechanism can be understood within the typical paradigm. In Fig. 2(a) we plot the beam 2D tune footprint $\rho\left(Q_{x}, Q_{y}\right)$ defined as

$$
\rho\left(Q_{x}, Q_{y}\right)=\sum_{i}\left|\tilde{x}_{i}\left(Q_{x}\right)\right|^{2}\left|\tilde{y}_{i}\left(Q_{y}\right)\right|^{2},
$$

where

$$
\tilde{x}_{i}\left(Q_{x}\right)=\frac{1}{C_{x i}} \int x_{i}(s) e^{i \omega_{0} Q_{x} s} d s
$$

is the Fourier transform of the particle $i$ horizontal displacement $x_{i}(s)$. An analogous definition is used for $\tilde{y}_{i}\left(Q_{y}\right)$. Since the Landau damping is determined by the tune density and is insensitive to the particles' amplitude per se, the constant $C_{x i}$ in Eq. (17) is used to normalize the spectral weight of each particle, Eq. (16), to one. As a consequence, the integrated $\rho\left(Q_{x}, Q_{y}\right)$ over the horizontal and the vertical frequencies is equal to the number of particles in the bunch. The space charge force shifts the particles' tunes to lower values. The tune depression is maximal at the bunch center, while the particles in the distribution tails have a much smaller tune shift. The satellite lines separated by $2 Q_{s}$ are a consequence of the modulation of the tune shift with the particle's longitudinal position [as Eq. (7) predicts]. The particles directly

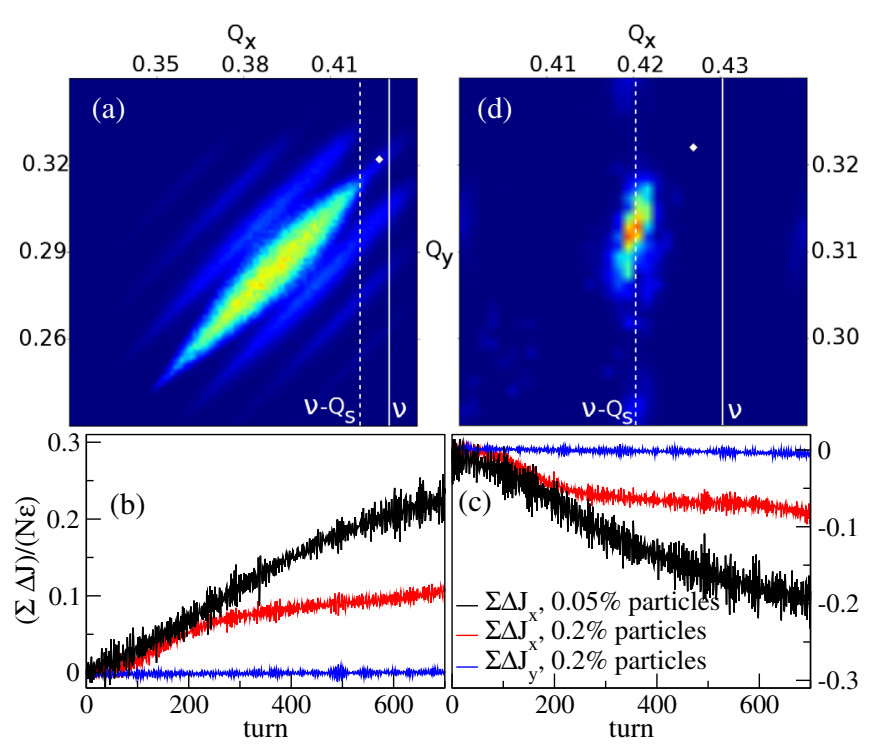

FIG. 2. (a) Bunch tune footprint at off-resonance for $q=7.94$. The white dot corresponds to the bare betatron tunes. (b) $\sum_{i \in S} \Delta J_{x}=\sum_{i \in S}\left(J_{x i}-J_{x i \text { initial }}\right)$ and $\sum_{i \in S} \Delta J_{y i}=\sum_{i \in S}\left(J_{y i}-\right.$ $\left.J_{y i \text { initial }}\right)$ of the $0.05 \%$ and $0.2 \%$ largest increasing energy particles versus turn number, normalized by the product of emittance and the number of the particles in the sum. (c) The same as (b) but for the largest decreasing energy particles. (d) Tune footprint for the $0.5 \%$ largest changing energy (increase and decrease) particles. The tunes are in the proximity of the coherent tune $Q_{x}=\nu-Q_{s}$. The color dimension scale in (a) and (d) differs by one order of magnitude.

responsible for the Landau damping are the ones which resonantly exchange energy with the mode. To select the LD-responsible particles we look for those having the largest change in their energy between the end and the beginning of the simulation. In Fig. 2(b) we plot

$$
\begin{aligned}
& \sum_{i \in S} \Delta J_{x}=\sum_{i \in S}\left(J_{x i}-J_{x i \text { initial }}\right) \quad \text { and } \\
& \sum_{i \in S} \Delta J_{y}=\sum_{i \in S}\left(J_{y i}-J_{y i \text { initial }}\right)
\end{aligned}
$$

where $J_{x i \text { initial }}$ and $J_{y i \text { initial }}$ represent the particle $i$ actions at the beginning of the simulation. The notation $S$ represents the subset of the $0.05 \%$ (black) or the $0.2 \%$ (red and blue) largest energy increase particles. A similar plot is shown in Fig. 2(c), but for the largest energy decrease particles. The values $0.05 \%$ and $0.2 \%$ are arbitrary chosen for the purpose of illustrating the properties of the LD-responsible particles. In the linear Landau damping theory, the energy of the LD-responsible particles increases linearly in a time interval $\delta t \approx 1 /|\delta \omega|$, where $\delta \omega$ is the frequency difference between the particle and the mode. As seen from Fig. 2(b), the time where $\sum \Delta J_{x}$ is increasing linearly is larger when the number of the particles in the summation is smaller, since a larger number in the summation implies particles with larger $|\delta \omega|$. Note that the chosen particles increase or 


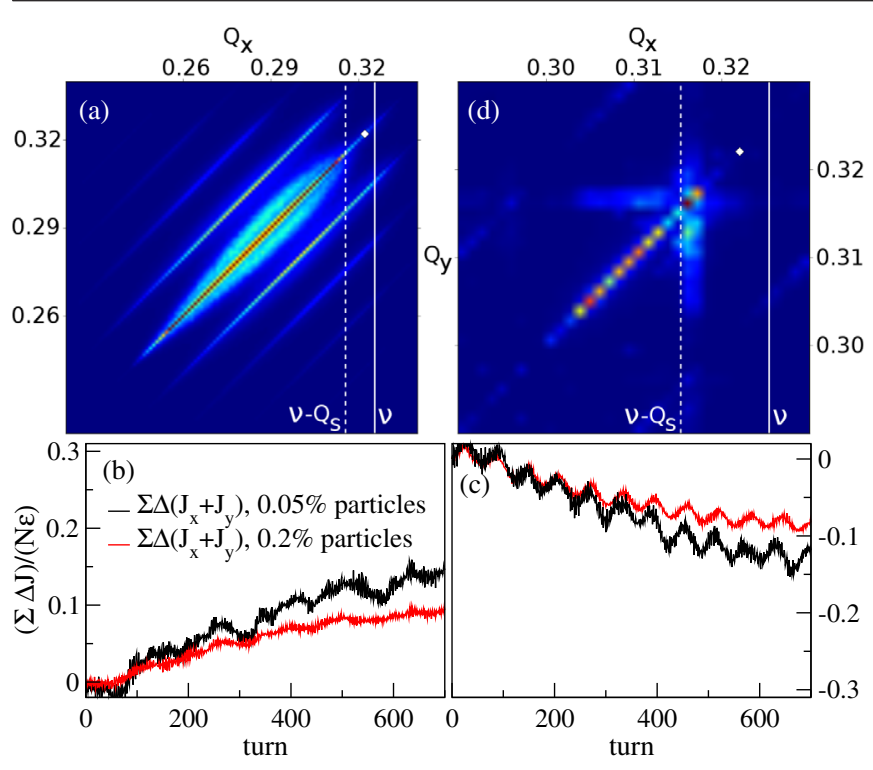

FIG. 3. (a) Bunch tune footprint at coupling resonance for $q=7.94$. The white dot corresponds to the bare betatron tunes. (b) $\sum_{i \in S} \Delta J_{s i}=\sum_{i \in S}\left(J_{s i}-J_{s i \text { initial }}\right)$ of the $0.05 \%$ and $0.2 \%$ largest increasing energy particles versus turn number, normalized by the product of emittance and the number of the particles in the sum. (c) The same as (b) but for the largest decreasing energy particles. (d) Tune footprint for the $0.5 \%$ largest changing energy (increase and decrease) particles. Large part of the spectral weight is along the resonance line $2 Q_{x}-2 Q_{y}=0$, with the horizontal tune well below $\nu-Q_{s}$. The color dimension scale in (a) and (d) differs by one order of magnitude.

decrease their action only in the horizontal plane, i.e., the plane where the mode is present. The 2D tune footprint of the $0.5 \%$ largest-energy changing particles (both increasing and decreasing) is shown in Fig. 2(d). As expected, since these particles are mode-resonant, their horizontal tune is in the vicinity of $\nu-Q_{s}$. Notice that higher $\nu-(2 n+1) Q_{s}$ harmonics spectral features do not appear to be significant in the tune footprint of the LD-responsible particles.

The spectral properties of the LD-responsible particles at the coupling resonance do not fit the typical Landau damping paradigm. The beam $2 \mathrm{D}$ tune footprint in Fig. 3(a) displays enhanced spectral weight along the coupling resonance line $2 Q_{x}-2 Q_{y}=0$, consequence of resonance trapping [in agreement with Eqs. (A45) and (A46)]. Satellite lines corresponding to the $2 Q_{s}$ harmonics can be observed too. We use the same largest energy change criterion to select the LD-responsible particles. Unlike the off-resonance case, the horizontal and vertical actions exhibit nonmonotonic change with turn number, since in the proximity of coupling resonance their magnitude oscillates between the planes. However, the transverse action sum $J_{s}$ of the LD-responsible particles displays a monotonic increase (decrease), as shown in Fig. 3(b) [Fig. 3(c)]. The interesting fact which points to a parametric damping mechanism is that the tune of most of these large energy changing particles is not in the vicinity of $\nu-Q_{s}$ as

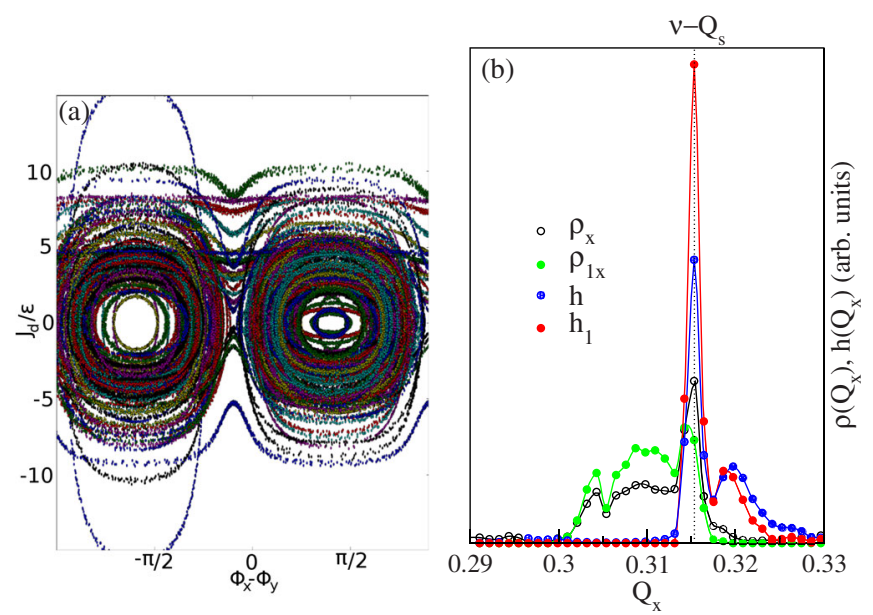

FIG. 4. Coupling resonance, $q=7.94$. (a) Poincare plots, $J_{d}$ versus $\Phi_{x}-\Phi_{y}$, for randomly selected particles belonging to the $0.5 \%$ largest changing energy particles. Most of these particles are trapped in the resonance islands. (b) The horizontal tune density $\rho\left(Q_{x}\right)$ (black), the $Q_{t}$ shifted tune density $h\left(Q_{x}\right)$ (blue) and the one-tune-per-particle tune density $\rho_{1}\left(Q_{x}\right)$ (green) with the corresponding $Q_{t}$ shifted tune density $h_{1}\left(Q_{x}\right)$ for the $0.5 \%$ largest changing energy particles. $h\left(Q_{x}\right)$ and $h_{1}\left(Q_{x}\right)$ are strongly peaked at the resonant mode tune $Q_{x}=\nu-Q_{s}$, showing modeparticle resonance via the $B J_{d} \bar{x}$ term [see Eq. (12)].

one would expect for LD-responsible particles. As shown in Fig. 3(d), there is a large spectral weight on the coupling resonance line which extends well below $Q_{x}=\nu-Q_{s}$.

Most of the large changing energy particles are trapped in resonance islands, as can be seen in Fig. 4(a) where the Poincare plots, $J_{d i}$ versus $\Phi_{x i}-\Phi_{y i}$ for $i \in S$, are shown. The phase coordinates are defined as

$$
\begin{aligned}
& \Phi_{x i}=\arctan \left(-\frac{\alpha_{x} x_{i}+\beta_{x} x_{i}^{\prime}}{x_{i}}\right), \\
& \Phi_{y i}=\arctan \left(-\frac{\alpha_{y} y_{i}+\beta_{y} y_{i}^{\prime}}{y_{i}}\right) .
\end{aligned}
$$

The Landau damping is, therefore, strongly influenced by the $J_{d}$ oscillations characterizing the coupling resonance trapped particles. The trapping frequency, $\omega_{0} Q_{t i}$, of the LD-responsible particles is determined from the Fourier spectrum of $J_{d i}(s)$.

The $Q_{t}$ satellites in the particles' tune spectra contribute to the spectral weight at the mode coherent tune $\nu-Q_{s}$ by $\approx 20 \%-25 \%$. This contribution favors the conventional damping mechanism via the $A \bar{x}$ coupling [see Eq. (12)]. To estimate the $Q_{t}$ satellites' spectral weight we compare the horizontal tune density

$$
\rho_{x}(Q)=\sum_{i \in S} \rho_{x i}(Q)=\sum_{i \in S}\left|\tilde{x}_{i}(Q)\right|^{2}
$$

and the one-tune-per-particle density

$$
\rho_{1 x}(Q)=\sum_{i \in S} \rho_{1 x i}(Q)=\sum_{i \in S} \delta\left(Q-\bar{Q}_{x i}\right)
$$


The sums in Eqs. (20) and (21) are restricted only to the number of the selected particles with the largest energy change. $\bar{Q}_{x i}$ is the tune of the largest spectral peak in the Fourier spectrum $\left|\tilde{x}_{i}(Q)\right|$. Unlike $\rho_{x}$, where all spectral features are present, $\rho_{1 x}$ assumes that every particle is characterized only by its main tune. The spectral weight difference between $\rho_{x}$ and $\rho_{1 x}$ at $\nu-Q_{s}$ measures the satellites contribution to the $A \bar{x}$ damping mechanism. In Fig. 4(b) $\rho_{x}$ and $\rho_{1 x}$ for the $0.5 \%$ largest changing energy particles are shown. Besides the peak at $\nu-Q_{s}$, a broad spectral feature at smaller frequency, unfavorable to the $A \bar{x}$ damping mechanism, is observed in both $\rho_{x}$ and $\rho_{1 x}$.

The other contribution of the $J_{d}$ oscillations to the damping is via the $B J_{d} \bar{x}$ term. The resonance condition is $Q_{x i}+Q_{t i}=\nu-Q_{s}$. We define $h(Q)$ as the tune density obtained by shifting each particle's horizontal tune by $Q_{t i}$, such

$$
\begin{aligned}
h(Q) & =\sum_{i \in S} h_{i}(Q)=\sum_{i \in S} \int \rho_{J d i}\left(Q^{\prime}\right) \rho_{x i}\left(Q-Q^{\prime}\right) d Q^{\prime} \\
& \approx \sum_{i \in S} \rho_{x i}\left(Q-Q_{t i}\right),
\end{aligned}
$$

where $\rho_{J d i}(Q)=\left|\tilde{J}_{d i}(Q)\right|^{2}$ is the Fourier spectrum of $J_{d i}(s)$. We define $h_{1}(Q)$ by replacing $\rho_{x i}$ with $\rho_{1 x i}$ in Eq. (22). As shown in Fig. 4(b), both $h(Q)$ and $h_{1}(Q)$ are strongly peaked at the coherent frequency $\nu-Q_{s}$ and do not display the broad spectral feature seen in $\rho_{x}(Q)$ and $\rho_{1 x}(Q)$ below $\nu-Q_{s}$. In fact the particles with the tune forming the broad spectral feature of $\rho_{1 x}(Q)$ have the main tune $\bar{Q}_{x i} \approx \nu-Q_{s}-Q_{t i}$, i.e., the tune required for resonance with the $B J_{d} \bar{x}$ coupling term. The broad feature seen in $h(Q)\left[h_{1}(Q)\right]$ spectrum above $\nu-Q_{s}$ corresponds to the spectral weight at $\nu-Q_{s}$ in the tune density $\rho_{x}(Q)$ $\left[\rho_{1 x}(Q)\right]$ when shifted with particle dependent $Q_{t i}$.

Compared to the off-resonance case, at coupling resonance the mode-particle coupling term $B J_{d} \bar{x}$ allows a larger number of particles to participate to the damping process. The conventional coupling does not favor particles with small longitudinal amplitudes, since they have a large tune shift which excludes them from the resonant exchange process with the mode. However, this impediment is not so restrictive for the resonant exchange via the $B J_{d} \bar{x}$ term, since the trapping frequency $Q_{t}$ is proportional to the charge density, thus also being large for small longitudinal amplitude particles and partially compensating for the large tune shift.

\section{DISCUSSIONS}

In our simulations we choose the initial amplitude excitation of the space charge mode to be small, of order of $10^{-3} \sigma_{x}$, where $\sigma_{x}$ is the beam horizontal rms size. As a consequence, the change in the beam shape and size are of the same order of magnitude. In a good approximation the

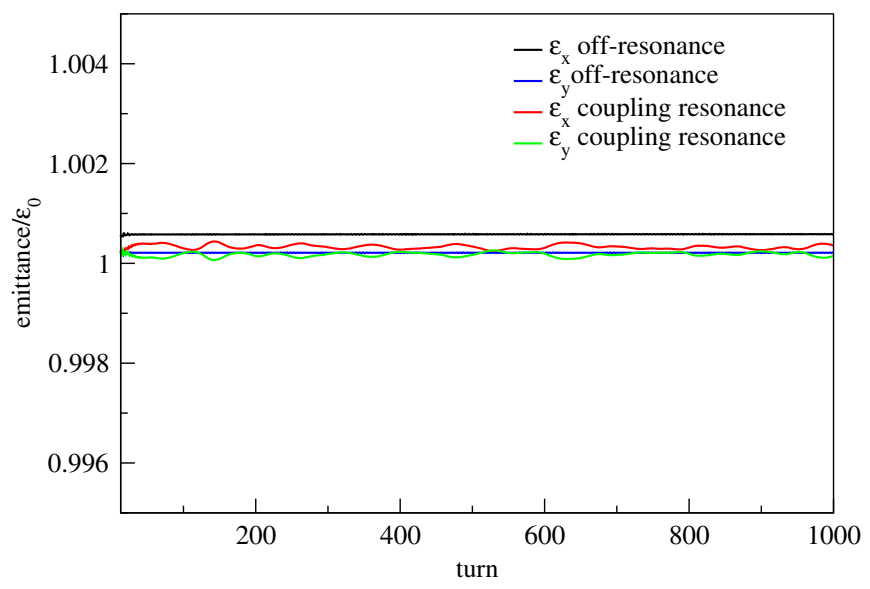

FIG. 5. The horizontal and the vertical emittances versus the turn number for the off-resonance and the coupling resonance cases for $q=7.94$. The emittances are nearly constant, the relative change being smaller than $5 \times 10^{-4}$.

beam size is constant. One may wonder about beam shape at the coupling resonance case, which, in general, is associated with the Montague's emittance exchange between transverse planes [10]. The Montague's emittance exchange occurs when the horizontal and the vertical emittances differs significantly from each other. In our case the initial beam distribution is chosen such that $\epsilon_{x}=\epsilon_{y} \equiv \epsilon_{0}$, and the emittance exchange is negligible. While at the particle level the coupling resonance is characterized by amplitude exchange between the transverse planes, the overall beam distribution changes very little. In Fig. 5 we plot the transverse emittances versus the turn number, both at the coupling resonance and in the offresonance case. The relative change of the horizontal and the vertical emittances are smaller than $5 \times 10^{-4}$.

While our simulations show enhanced damping at the coupling resonance it is important to address the damping behavior when moving away from this point. We investigate the damping as a function of the bare betatron tunes difference $\Delta Q_{0}=Q_{0 x}-Q_{0 y}$. In Fig. 6 we plot the Landau damping of the horizontal first space charge mode versus $\Delta Q_{0} / Q_{s}$ for different values of the space charge parameter and the synchrotron tune. The width of the enhanced damping region scales linearly with the space charge tune shift, $W \approx 0.3 Q_{s c \max }$, where $W$ is defined as the width at half maximum. The enhanced damping region is asymmetric with respect the to $\Delta Q_{0}=0$, extending predominantly $(\approx 80 \%)$ on the negative side of $\Delta Q_{0}$. We notice two maxima of the damping rate in the enhanced damping region. One is at coupling resonance $\Delta Q_{0}=0$ and the other at $-\Delta Q_{0}=M$. The value of $M$ increases slightly increasing $q$ as shown in the inset of Fig. 6. Our preliminary results indicate that the $-\Delta Q_{0}=M$ maximum and the asymmetry of the enhanced damping region are related to a different modulation of the mode-particle coupling. Those results will be addressed in detail in a future publication. 


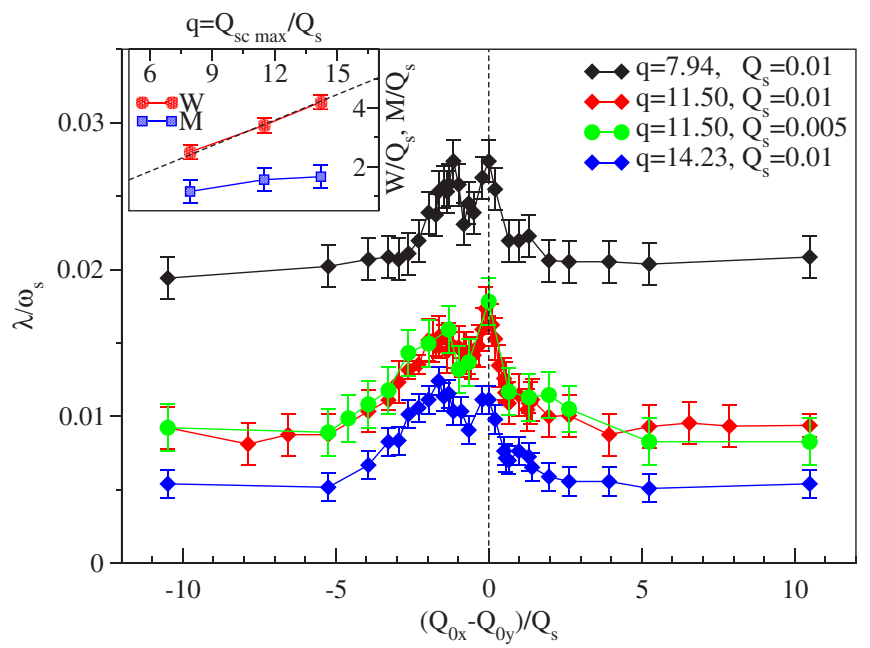

FIG. 6. Landau damping rate $\frac{\lambda}{\omega_{s}}$ of the horizontal first space charge mode versus $\Delta Q_{0} / Q_{s}=\left(Q_{0 x}-Q_{0 y}\right) / Q_{s}$ for different values of the space charge parameter $q$ and the synchrotron tune $Q_{s}$ where $\omega_{s}=\omega_{0} Q_{s}$ is the synchrotron frequency. The width of the enhanced damping region in the proximity of coupling resonance $W$, defined at half maximum, scales linearly with the space charge tune shift, $W \approx 0.3 Q_{s c \text { max }}$, as shown in the inset. The enhanced damping region is asymmetric with respect the to $\Delta Q_{0}=0$, extending predominantly on the negative side of $\Delta Q_{0}$. Two maxima of the damping rate can be noticed, one at $\Delta Q_{0}=0$ and the other one at $\Delta Q_{0} \approx-M$.

\section{CONCLUSIONS}

A novel Landau damping mechanism, driven by the modulation of the mode-particle coupling is introduced. Numerical simulations using SYNERGIA with the DMD method show the existence of this mechanism in bunched beams in the proximity of coupling resonance. The properties of the first space charge mode are calculated for a Gaussian bunch propagating through an OFORODO lattice. The off-resonance and the coupling resonance cases are compared. While the space charge mode's tune and shape are nearly the same, the Landau damping is approximately a factor of 2 larger at coupling resonance in the strong space charge regime. In the off-resonance case the damping mechanism can be understood within the conventional paradigm. The damping is caused by the resonant energy exchange between the mode and the particles with an incoherent tune equal to the mode's tune shifted by $Q_{s}$. At coupling resonance a large number of particles are trapped around the stable points. Their transverse actions are oscillating with a particle dependent trapping frequency $\omega_{0} Q_{t}$. The spectral properties of the trapped particles with large energy exchange reveal that their tune is additionally shifted from the mode's coherent tune by $Q_{t}$, supporting the parametric Landau damping mechanism.

\section{ACKNOWLEDGMENTS}

This manuscript has been authored by Fermi Research Alliance, LLC under Contract No. DE-AC02-07CH11359 with the U.S. Department of Energy, Office of Science, Office of High Energy Physics. Synergia development is partially supported through the ComPASS project, funded through the Scientific Discovery through Advanced Computing program in the DOE Office of High Energy Physics. An award of computer time was provided by the Innovative and Novel Computational Impact on Theory and Experiment (INCITE) program. This research used resources of the Argonne Leadership Computing Facility, which is a DOE Office of Science User Facility supported under Contract No. DE-AC02-06CH11357.

\section{APPENDIX: TUNE SHIFT AND MODE-PARTICLE COUPLING}

The space charge induced tune shift and the coupling of the space charge collective modes to the incoherent motion of the particles are dependent on the particles amplitudes. The interpretation of our numerical results is based on this assumption. For realistic beams it is difficult to derive analytical expressions for the tune shift and the modeparticle coupling. In this appendix we calculate these expressions using a simply model. Despite the simplifying assumptions, the derivations provide insight on the dependence of the tune shift and the mode-particle coupling on the particles properties.

We consider a 2D model with transverse degrees of freedom

$$
H=\frac{p_{x}^{2}}{2}+\frac{1}{2} \omega_{0 x}^{2} x^{2}+\frac{p_{y}^{2}}{2}+\frac{1}{2} \omega_{0 y}^{2} y^{2}+V(x, y),
$$

were $V(x, y)$ is the space charge interaction part of the Hamiltonian.

\section{Off-resonance case}

We will introduce two different approaches for particle's frequency calculation. In the first approach one writes the Hamiltonian in the canonical action-angle coordinates

$$
H=\omega_{0 x} J_{x}+\omega_{0 y} J_{y}+V\left(J_{x}, J_{y}, \Phi_{x}, \Phi_{y}\right),
$$

where

$$
x=\sqrt{\frac{2 J_{x}}{\omega_{0 x}}} \sin \Phi_{x}, \quad p_{x}=\sqrt{2 J_{x} \omega_{0 x}} \cos \Phi_{x} .
$$

Analogous transformations are used for $y$ and $p_{y}$. Since the space charge potential contains only terms with even powers of $x$ and $y$, it can be written as

$$
\begin{aligned}
& V\left(J_{x}, J_{y}, \Phi_{x}, \Phi_{y}\right) \\
& =V_{0}\left(J_{x}, J_{y}\right)+V_{1}\left(J_{x}, J_{y}, \cos 2 m \Phi_{x}, \cos 2 n \Phi_{y}\right), m, n>0 .
\end{aligned}
$$


$V_{1}$ contains only high harmonics terms proportional to $\cos 2 m \Phi_{x}$ and/or $\cos 2 n \Phi_{y}$. Away from resonances (see Appendix A 2 for a discussion of the coupling resonance), the contribution of these terms averages to zero in time. $V_{1}$ can be neglected in the first order of perturbation theory. The particle's horizontal frequency is given by

$$
\omega_{x}=\dot{\Phi}_{x}=\frac{\partial\left(H_{0}+V_{0}\right)}{\partial J_{x}}=\omega_{0 x}+\frac{\partial V_{0}}{\partial J_{x}} .
$$

The frequency shift is

$$
\delta \omega_{x}\left(J_{x}, J_{y}\right)=\omega_{0 x}-\omega_{x}=-\frac{\partial V_{0}}{\partial J_{x}}\left(J_{x}, J_{y}\right) .
$$

Analogous equations can be written for the vertical frequency.

The second approach for the particle's frequency calculation starts from the equation of motion and is based on the Lindstedt-Poincare perturbation theory [24] for nonlinear equations. The horizontal displacement obeys

$$
\ddot{x}+\omega_{0 x}^{2} x=F(x, y),
$$

where

$$
F(x, y)=-\frac{\partial V}{\partial x}(x, y)
$$

is the space charge force considered to be a perturbation. In the first order of perturbation theory, only the terms in $F(x, y)$ which oscillate with a frequency close to $\omega_{x}$ are relevant for the particle dynamics. To isolate these terms we write $x$ and $y$ in the action-angle coordinates. $F(x, y)$ contains terms with odd powers of $x$ and even powers of $y$ and it can be written as

$$
\begin{aligned}
F(x, y)= & F_{0}\left(J_{x}, J_{y}\right) \sin \Phi_{x} \\
& +F_{1}\left(J_{x}, J_{y}, \sin (2 m+1) \Phi_{x}, \cos 2 n \Phi_{y}\right) \\
& m, n>0 .
\end{aligned}
$$

$F_{1}$ can be neglected since it contains only higher harmonics of $\omega_{x}=\dot{\Phi}_{x}$. Equation (A7) reduces to

$$
\ddot{x}+\left(\omega_{0 x}^{2}-F_{0}\left(J_{x}, J_{y}\right) \sqrt{\frac{\omega_{0 x}}{2 J_{x}}}\right) x=0,
$$

and the horizontal tune shift is

$$
\delta \omega_{x}\left(J_{x}, J_{y}\right)=F_{0}\left(J_{x}, J_{y}\right) \frac{1}{2 \sqrt{2 J_{x} \omega_{0 x}}} .
$$

Using Eqs. (A3) and (A8) one can check that Eq. (A6) and Eq. (A11) agree with each other.
To estimate the mode-particle coupling we will use the equation of motion. We consider a transverse mode which displaces the beam center infinitesimally by $\bar{x}$. Assuming the rigid-slice approximation, the space charge potential in this case is

$$
V(x-\bar{x}, y)=V(x, y)-\frac{\partial V(x, y)}{\partial x} \bar{x} .
$$

The equation of motion reads

$$
\ddot{x}+\omega_{0 x}^{2} x=F(x, y)-\frac{\partial F}{\partial x}(x, y) \bar{x} .
$$

The mode-particle coupling is given by the last term in Eq. (A13). In order to address the Landau damping mechanism, one has to investigate the parts in the $\frac{\partial F}{\partial x}(x, y) \bar{x}$ term which are in resonance with the particle displacement $x$. Let us assume in this section that the mode frequency $\omega_{c}$ is close to the particle's one $\omega_{x}$, as it happens for the conventional Landau mechanism. Since $\frac{\partial F}{\partial x}(x, y)$ contains only even powers of $x$ and $y$ one can write

$$
\begin{aligned}
\frac{\partial F}{\partial x}(x, y)= & G_{0}\left(J_{x}, J y\right)+G_{1}\left(J_{x}, J_{y}\right) \cos 2 \Phi_{x} \\
& +G_{2}\left(J_{x}, J_{y}, \cos 2 m \Phi_{x}, \cos 2 n \Phi_{y}\right) \\
& m, n>0 .
\end{aligned}
$$

Neglecting the high harmonics, the equation of motion can be written as

$$
\begin{aligned}
& \ddot{x}+\left[\omega_{0 x}-\delta \omega_{x}\left(J_{x}, J_{y}\right)\right]^{2} x \\
& \approx-G_{0}\left(J_{x}, J y\right) \bar{x}+\frac{M}{2} G_{1}\left(J_{x}, J_{y}\right) \sin \left(2 \Phi_{x}-\omega_{c} t\right) .
\end{aligned}
$$

Here $M$ is the mode amplitude, $\bar{x}=M \sin \omega_{c} t$, and $\delta \omega_{x}\left(J_{x}, J_{y}\right)$ is given by Eq. (A11). Since $\sin \left(2 \Phi_{x}-\omega_{c} t\right) \approx$ $\sin \left(\omega_{c} t\right)$ when $\omega_{c} \approx \omega_{x}$ and the oscillations of $x$ and $\bar{x}$ are in phase [25], one can write the equation of motion as

$$
\ddot{x}+\left[\omega_{0 x}-\delta \omega_{x}\left(J_{x}, J_{y}\right)\right]^{2} x=-K\left(J_{x}, J_{y}\right) \bar{x},
$$

with the mode-particle coupling given by

$$
K\left(J_{x}, J_{y}\right)=G_{0}\left(J_{x}, J_{y}\right)-\frac{1}{2} G_{1}\left(J_{x}, J_{y}\right) .
$$

We will end this section with an example. Let us consider a space charge potential

$$
V(x)=\alpha x^{4} .
$$

This is not a realistic potential but can be viewed as a term in the Taylor's expansion of the real potential. The space charge force is 


$$
\begin{aligned}
F(x) & =-4 \alpha x^{3}=-4 \alpha \frac{2 J_{x}}{\omega_{0 x}} \sqrt{\frac{2 J_{x}}{\omega_{0 x}}} \sin ^{3} \Phi \\
& =-4 \alpha \frac{2 J_{x}}{\omega_{0 x}} \sqrt{\frac{2 J_{x}}{\omega_{0 x}}}\left(\frac{3}{4} \sin \Phi_{x}-\frac{1}{4} \sin 3 \Phi_{x}\right) .
\end{aligned}
$$

Therefore

$$
F_{0}\left(J_{x}\right)=-\alpha \frac{6 J_{x}}{\omega_{0 x}} \sqrt{\frac{2 J_{x}}{\omega_{0 x}}},
$$

and according to Eq. (A11) the tune shift is

$$
\delta \omega_{x}\left(J_{x}\right)=-3 \alpha \frac{J_{x}}{\omega_{0 x}^{2}} .
$$

For the mode-particle coupling we have

$\frac{\partial F}{\partial x}(x, y)=-12 \alpha x^{2}=-12 \alpha \frac{J_{x}}{\omega_{0 x}}\left(1-\cos 2 \Phi_{x}\right)$,

implying

$$
K\left(J_{x}\right)=-18 \alpha \frac{J_{x}}{\omega_{0 x}}=3 \times 2 \omega_{0 x} \delta \omega_{x}\left(J_{x}\right) .
$$

The factor of 3 in front of the right-hand side (r.h.s.) of the Eq. (A23) arises from the derivative of $F(x, y)$ with respect to $x$. A space charge force term $F(x) \propto x^{2 n+1}$, implies $K\left(J_{x}\right)=(2 n+1) \times 2 \omega_{0 x} \delta \omega_{x}\left(J_{x}\right)$. In the linear regime where $F(x) \propto x$, valid for small amplitude oscillations, $K\left(J_{x}\right)=2 \omega_{0 x} \delta \omega_{x}\left(J_{x}\right)$.

Note that Eq. (4) proposed to describe the particles motion in Sec. III, is a generalization of Eq. (A16) to include longitudinal degrees of freedom.

\section{Transverse coupling resonance for round beams}

The resonance effects on beam particles dynamics are treated extensively in the literature. However the question of how the resonance effects can influence the modeparticle coupling and implicitly the Landau damping mechanism has not been addressed. In this section, using a simplified space charge potential, we will discuss the mode-particle coupling in the proximity of transverse coupling resonance given by $2 \omega_{x}-2 \omega_{y}=0$.

To illustrate the calculation of tune shift and modeparticle coupling near coupling resonance we consider an example where the space charge potential is rotationally symmetric and given by

$$
V(x, y)=\alpha\left(x^{2}+y^{2}\right)^{2} .
$$

In the proximity of the coupling resonance it is easier to calculate the particles dynamics with a Hamiltonian formalism than by using the equation of motion. For the calculation of the frequency shift we transform the Hamiltonian to action-angle coordinates

$$
\begin{aligned}
H= & \omega_{0 x} J_{x}+\omega_{0 y} J_{y}+\frac{3 \alpha}{2}\left(\frac{J_{x}^{2}}{\omega_{0 x}^{2}}+\frac{J_{y}^{2}}{\omega_{0 y}^{2}}\right) \\
& +2 \alpha \frac{J_{x} J_{y}}{\omega_{0 x} \omega_{0 y}}\left(1+\frac{1}{2} \cos \left(2 \Phi_{x}-2 \Phi_{y}\right)\right) .
\end{aligned}
$$

We neglect the high harmonics terms, as discussed in Sec. A 1. Assuming proximity of the coupling resonance the term proportional to $\cos \left(2 \Phi_{x}-2 \Phi_{y}\right)$ is retained.

We proceed with a canonical transformation using the generating functional

$F\left(\Phi_{x}, \Phi_{y}, I_{x}, I_{y}\right)=\left(\Phi_{x}-\bar{\omega} t\right) I_{x}+\left(\Phi_{y}-\bar{\omega} t\right) I_{y}$,

with $\bar{\omega}=\frac{1}{2}\left(\omega_{0 x}+\omega_{0 y}\right)$. It leads to the Hamiltonian

$$
\begin{aligned}
\tilde{H}= & \Delta \omega\left(-I_{x}+I_{y}\right)+\frac{3 \alpha}{2}\left(\frac{I_{x}^{2}}{\omega_{0 x}^{2}}+\frac{I_{y}^{2}}{\omega_{0 y}^{2}}\right) \\
& +2 \alpha \frac{I_{x} I_{y}}{\omega_{0 x} \omega_{0 y}}\left(1+\frac{1}{2} \cos \left(2 \varphi_{x}-2 \varphi_{y}\right)\right),
\end{aligned}
$$

where

$$
\begin{gathered}
\Delta \omega=\frac{1}{2}\left(\omega_{0 y}-\omega_{0 x}\right) \\
\varphi_{x}=\Phi_{x}-\bar{\omega} t, \quad J_{x}=I_{x} \\
\varphi_{y}=\Phi_{y}-\bar{\omega} t, \quad J_{y}=I_{y} \\
\tilde{H}=H-\bar{\omega}\left(I_{x}+I_{y}\right) .
\end{gathered}
$$

A subsequent canonical transformation using the generating functional

$F\left(\varphi_{x}, \varphi_{y}, J_{s}, J_{d}\right)=\frac{1}{2} J_{s}\left(\varphi_{x}+\varphi_{y}\right)+\frac{1}{2} J_{d}\left(\varphi_{x}-\varphi_{y}\right)$,

leads to the Hamiltonian

$$
\begin{aligned}
H= & -\Delta \omega J_{d}+\alpha\left[a_{s} J_{s}^{2}+a_{d} J_{d}^{2}+b J_{s} J_{d}\right. \\
& \left.+k\left(J_{s}^{2}-J_{d}^{2}\right) \cos \left(4 \varphi_{d}\right)\right]
\end{aligned}
$$

where the canonical variables are

$$
\begin{aligned}
\varphi_{s} & =\frac{1}{2}\left(\varphi_{x}+\varphi_{y}\right), & J_{s} & =I_{x}+I_{y} \\
\varphi_{d} & =\frac{1}{2}\left(\varphi_{x}-\varphi_{y}\right), & J_{d} & =I_{x}-I_{y}
\end{aligned}
$$


and

$$
\begin{aligned}
a_{s} & =\frac{3}{8}\left(\frac{1}{\omega_{0 x}^{2}}+\frac{1}{\omega_{0 y}^{2}}+\frac{4}{3 \omega_{0 x} \omega_{0 y}}\right) \\
a_{d} & =\frac{3}{8}\left(\frac{1}{\omega_{0 x}^{2}}+\frac{1}{\omega_{0 y}^{2}}-\frac{4}{3 \omega_{0 x} \omega_{0 y}}\right) \\
b & =\frac{3}{4}\left(\frac{1}{\omega_{0 x}^{2}}-\frac{1}{\omega_{0 y}^{2}}\right) \\
k & =\frac{1}{4} \frac{1}{\omega_{0 x} \omega_{0 y}} .
\end{aligned}
$$

Since the Hamiltonian is independent on $\varphi_{s}$, it follows that $J_{s}$ is a constant of motion.

The stable point $\left(\varphi_{d}^{*}, J_{d}^{*}\right)$ is given by the equations $\dot{J}_{d}=0, \dot{\varphi}_{d}=0$ which lead to

$$
\cos \left(4 \varphi_{d}^{*}\right)=-1, \quad J_{d}^{*}=\frac{\Delta \omega \bar{\omega}^{2}}{\alpha},
$$

up to the first order in $\Delta \omega$. The particle dynamics around the stable point is described by the effective Hamiltonian

$$
H_{\mathrm{eff}}=\frac{\alpha}{2 \bar{\omega}^{2}} \delta J_{d}^{2}+2 \frac{\alpha}{\bar{\omega}^{2}}\left(J_{s}^{2}-J_{d}^{* 2}\right) \delta \varphi_{d}^{2}
$$

where $\delta J_{d}=J_{d}-J_{d}^{*}, \delta \varphi_{d}=\varphi_{d}-\varphi_{d}^{*}$. The oscillation frequency of $J_{d}$ in the proximity of the coupling resonance stable point is therefore

$$
\omega_{t}=\frac{2 \alpha}{\bar{\omega}^{2}} \sqrt{\left(J_{s}^{2}-J_{d}^{* 2}\right)} .
$$

Considering that

$$
\begin{aligned}
& \dot{\varphi}_{s}=2 \alpha\left(a_{s}+k \cos \left(4 \varphi_{d}\right)\right) J_{s}=2 \frac{\alpha}{\bar{\omega}^{2}} J_{s} \\
& \dot{\varphi}_{d}=\frac{\alpha}{\bar{\omega}^{2}} \delta J_{d}
\end{aligned}
$$

the particle tunes are

$$
\begin{aligned}
& \omega_{x} \approx \dot{\Phi}_{x}=\dot{\varphi}_{s}+\dot{\varphi}_{d}+\bar{\omega}=2 \frac{\alpha}{\bar{\omega}^{2}} J_{s}+\bar{\omega}+\frac{\alpha}{\bar{\omega}^{2}}\left(J_{d}-J_{d}^{*}\right) \\
& \omega_{y} \approx \dot{\Phi}_{y}=\dot{\varphi}_{s}-\dot{\varphi}_{d}+\bar{\omega}=2 \frac{\alpha}{\bar{\omega}^{2}} J_{s}+\bar{\omega}-\frac{\alpha}{\bar{\omega}^{2}}\left(J_{d}-J_{d}^{*}\right) .
\end{aligned}
$$

Note that in the frequency space the main spectral feature of the trapped particle resides on the line $\omega_{x}=\omega_{y}$. The oscillation of $J_{d}$ yields satellite features spaced by harmonics of the trapping frequency $\omega_{t}$.
The frequency shift is given by

$$
\begin{aligned}
& \delta \omega_{x}\left(J_{s}, J_{d}\right)=\omega_{0 x}-\omega_{x}=\Delta \omega+2 \frac{\alpha}{\bar{\omega}^{2}} J_{s}+\frac{\alpha}{\bar{\omega}^{2}}\left(J_{d}-J_{d}^{*}\right) \\
& \delta \omega_{y}\left(J_{s}, J_{d}\right)=\omega_{0 y}-\omega_{y}=-\Delta \omega+2 \frac{\alpha}{\bar{\omega}^{2}} J_{s}-\frac{\alpha}{\bar{\omega}^{2}}\left(J_{d}-J_{d}^{*}\right) .
\end{aligned}
$$

In the presence of a transverse mode, in the rigid-slice approximation, the space charge potential is

$$
V(x, y)=\alpha\left((x-\bar{x})^{2}+y^{2}\right)^{2} .
$$

The calculation of the mode-particle coupling reduces to analyzing the force acting on the particle. The equation of motion reads,

$\ddot{x}+\omega_{0 x}^{2} x=-4 \alpha x\left(x^{2}+y^{2}\right)+12 \alpha x^{2} \bar{x}+4 \alpha y^{2} \bar{x}$.

The displacement $\bar{x}$ is small and does not affect the incoherent particle motions in the first order approximation. Equation (A50) yields the mode-particle coupling written in action-angle coordinates as

$$
\begin{aligned}
-K \bar{x}= & \left(12 \alpha x^{2}+4 \alpha y^{2}\right) \bar{x} \\
= & {\left[12 \alpha \frac{\left(J_{s}+J_{d}\right)}{\omega_{0 x}} \sin ^{2} \Phi_{x}+4 \alpha \frac{\left(J_{s}-J_{d}\right)}{\omega_{0 y}} \sin ^{2} \Phi_{y}\right] \bar{x} } \\
= & 2 \alpha J_{s}\left[\frac{3}{\omega_{0 x}}\left(1-\cos 2 \Phi_{x}\right)+\frac{1}{\omega_{0 y}}\left(1-\cos 2 \Phi_{y}\right)\right] \bar{x} \\
& +2 \alpha J_{d}\left[\frac{3}{\omega_{0 x}}\left(1-\cos 2 \Phi_{x}\right)-\frac{1}{\omega_{0 y}}\left(1-\cos 2 \Phi_{y}\right)\right] \bar{x} .
\end{aligned}
$$

Because $J_{s}$ is constant, the first term in the r.h.s. of Eq. (A51) is resonant with $x$ when $\omega_{x} \approx \omega_{y} \approx \omega_{c}$, as expected by a conventional Landau damping mechanism. A similar reasoning with the one discussed in Appendix A 1 can be used to calculate the mode-particle coupling. However, when analyzing the terms resonant with $x$ one has to take into account that, according to Eq. (A40), the trapped particles phases satisfy $2 \Phi_{x}-2 \Phi_{y}=(2 k+1) \pi$. Doing that, one gets for the coupling term proportional to $J_{s}$,

$$
K_{1} \bar{x}=-J_{s}\left(\frac{9 \alpha}{\omega_{0 x}}+\frac{\alpha}{\omega_{0 y}}\right) \bar{x} \approx-\frac{10 \alpha J_{s}}{\omega_{0 x}} \bar{x} .
$$

On the other hand, since $J_{d}$ is oscillating with the frequency $\omega_{t}$, the term in the r.h.s. of Eq. (A51) proportional to $J_{d}$ is resonant with $x$ when $\omega_{x} \approx \omega_{y} \approx \omega_{c}-\omega_{t}$. Assuming that $J_{d}=\left|J_{d}\right| \cos \omega_{t} t, \quad \bar{x}=M \sin \omega_{c} t \quad$ and $2 \Phi_{x}-2 \Phi_{y}=(2 k+1) \pi$, the mode-particle coupling proportional to $J_{d}$ can be written as 


$$
K_{2} \bar{x}=-J_{d}\left(\frac{15 \alpha}{2 \omega_{0 x}}-\frac{3 \alpha}{2 \omega_{0 y}}\right) \bar{x} \approx-\frac{6 \alpha J_{d}}{\omega_{0 x}} \bar{x}
$$

The equation of motion in the proximity of the coupling resonance for our simplified Hamiltonian is

$$
\begin{aligned}
\ddot{x}+\left(\omega_{0 x}-\delta \omega_{x}\left(J_{s}, J_{d}\right)\right)^{2} x & =-\left(K_{1}+K_{2}\right) \bar{x} \\
& =\frac{10 \alpha}{\omega_{0 x}} J_{s} \bar{x}+\frac{6 \alpha}{\omega_{0 x}} J_{d} \bar{x} .
\end{aligned}
$$

The equation (12) proposed to described the motion of particles in the proximity of coupling resonance in Sec. III is a generalization of Eq. (A54) to include higher order terms in the space charge potential and, as well, longitudinal degrees of freedom.

[1] L. Landau, Oscillations of electron plasma, J. Phys. USSR 10, 25 (1946).

[2] J. Gareyte, J. P. Koutchouk, and F. Ruggiero, Report No. CERN-LHC-PROJECT-REPORT-091, 1997.

[3] M. Blaskiewicz, Fast head-tail instability with space charge, Phys. Rev. ST Accel. Beams 1, 044201 (1998).

[4] A. Burov, Head-tail modes for strong space charge, Phys. Rev. ST Accel. Beams 12, 044202 (2009); 12, 109901(E) (2009).

[5] V. Balbekov, Transverse modes of a bunched beam with space charge dominated impedance, Phys. Rev. ST Accel. Beams 12, 124402 (2009).

[6] V. Kornilov and O. Boine-Frankenheim, Transverse modes of a bunched beam with space charge dominated impedance, Phys. Rev. ST Accel. Beams 13, 114201 (2010).

[7] V. Kornilov and O. Boine-Frankenheim, Thresholds of the Head-Tail Instability in Bunches with Space Charge, in Proceedings of the 54th ICFA Advanced Beam Dynamics Workshop on High-Intensity, High Brightness and High Power Hadron Beams, East-Lansing, MI, USA (2014), http://epaper.kek.jp/HB2014.

[8] A. Macridin, A. Burov, E. Stern, J. Amundson, and P. Spentzouris, Simulation of transverse modes with their intrinsic Landau damping for bunched beams in the presence of space charge, Phys. Rev. ST Accel. Beams 18, 074401 (2015).
[9] Elias Metral, Ph.D. Thesis, CERN-THESIS, 1999-074.

[10] B. W. Montague, CERN Report No. 68-38, 1968.

[11] Synergia2, Accelerator Modeling, https://cdcvs.fnal.gov/ redmine/projects/synergia2/wiki.

[12] J. Amundson, P. Spentzouris, J. Qiang, and R. Ryne, Synergia: An accelerator modeling tool with 3-D space charge, J. Comp. Physiol. 211, 229 (2006).

[13] P. J. Schmid, Dynamic mode decomposition of numerical and experimental data, J. Fluid Mech. 656, 5 (2010).

[14] C. W. Rowley, I. Mezić, S. Bagheri, P. Schlatter, and D. S. Henningson, Spectral analysis of nonlinear flows, J. Fluid Mech. 641, 115 (2009).

[15] J. H. Tu, C. W. Rowley, D. M. Luchtenburg, S. L. Brunton, and J. N. Kutz, On dynamic mode decomposition: Theory and applications, J. Comp. Nonlin. Dyn. 1, 391 (2014).

[16] K. K. Chen, J. H. Tu, and C. W. Rowley, Variants of dynamic mode decomposition: Boundary condition, koopman, and fourier analyses, J. Nonlinear Sci. 22, 887 (2012).

[17] A. Chao, Physics of Collective Beam Instabilities in High Energy Accelerators (John Wiley \& Sons, Inc., New York, 1993), Eq 5.2.

[18] D. Mohl and H. Schonauer, Landau damping by non-linear space-charge forces and octupoles, in Proceedings of the IXth International Conference on High Energy Accelerators, Stanford (Stanford Linear Accelerator Center, Stanford, 1974), p. 380, http://www.slac.stanford.edu/ pubs/slacreports/reports16/slac-r-839-c.pdf.

[19] H. Yoshida, Construction of higher order symplectic integrators, Phys. Lett. A 150, 262 (1990).

[20] R. W. Hockney and J. W. Eastwood, Computer Simulation Using Particles (McGraw-Hill, New York, 1981).

[21] See Ref. [15] and the references therein.

[22] B. W. Brunton, L. A. Johnson, J. G. Ojemann, and J. Nathan Kutz, Extracting spatial-temporal coherent patterns in large-scale neural recordings using dynamic mode decomposition, J. Neurosci. Methods 258, 1 (2016).

[23] J. Grosek and J. Nathan Kutz, Dynamic mode decomposition for real-time background/foreground separation in video, arXiv:1404.7592.

[24] A. H. Nayfeh, Perturbation Methods (WILEY-VCH Valag $\mathrm{GmbH} \&$ Co. KGaA, New York, 2000), ch. 3.1.1

[25] For the Landau damping mechanism only the part of the solution in phase with the driving force is relevant, see chapter 5 in [17]. 